شناسايى زنهاى كنتر كننده صفات كياهجِهاى در جمعيت نوتر كيب برنج ايرانى تحت تنث خشكى

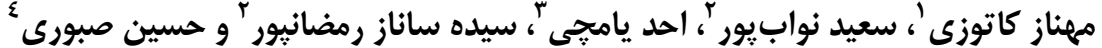

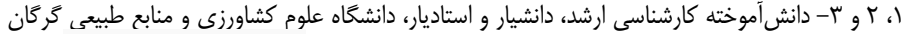

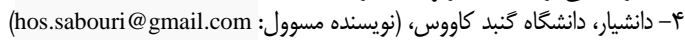 \\ تاريخ يذيرش: 9F/N/9

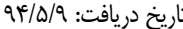

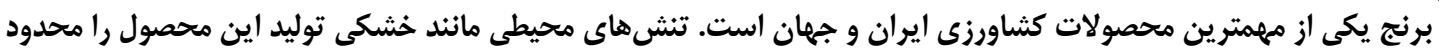

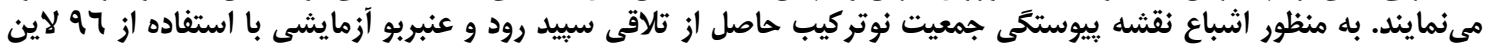

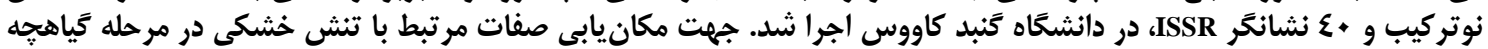

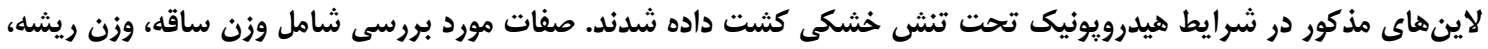

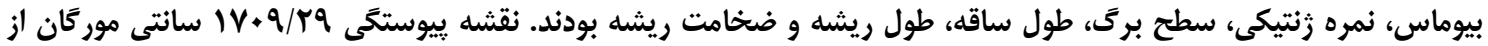

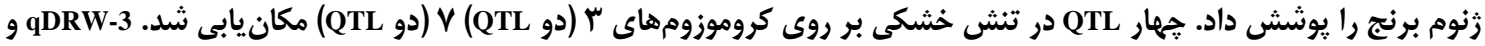

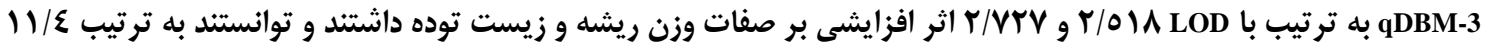

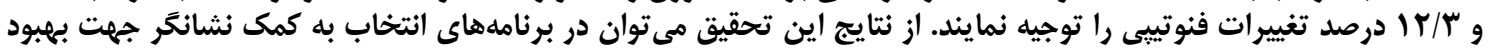

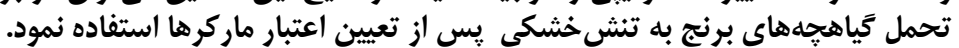

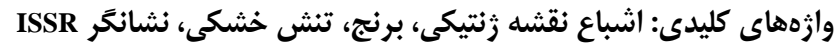

روى حجم ريشه تاثيركذار بود. همجنين نتايج اين تحقيق

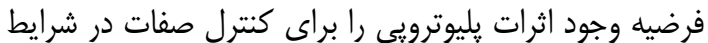

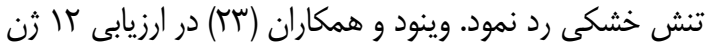

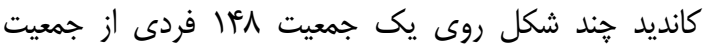

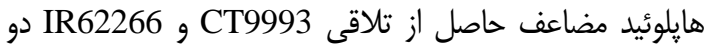

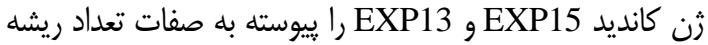

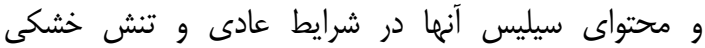

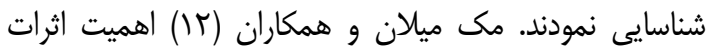

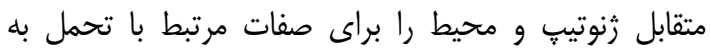

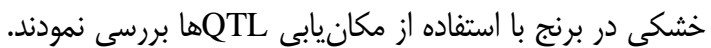

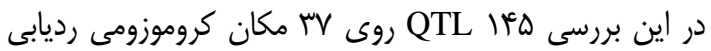

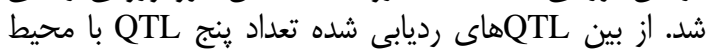

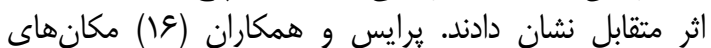

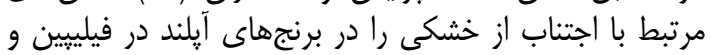

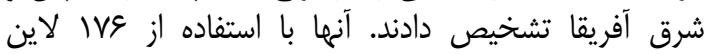

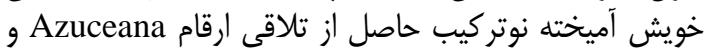

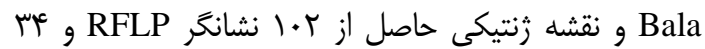

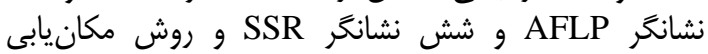

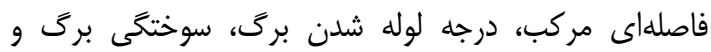

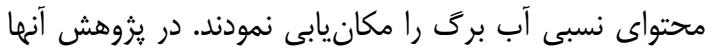

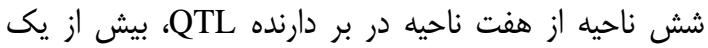

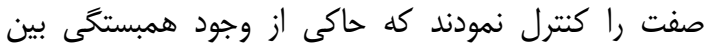

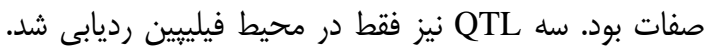

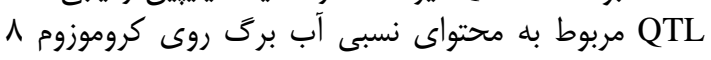

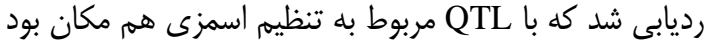

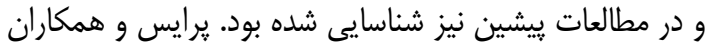

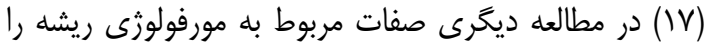
در جمعيت لاين خويش آميخته نوتركيب حاصل به از تلاقى رلى
مقدمه

برنج به عنوان يك گياه قادر به رشد در شرايط غرقابى،

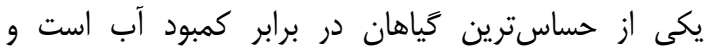

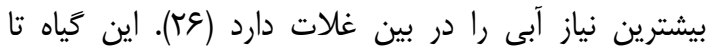

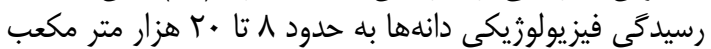

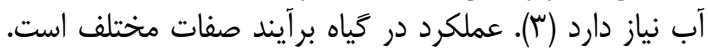

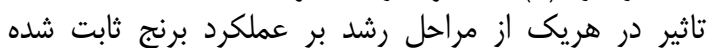

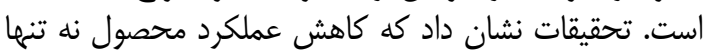

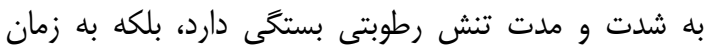

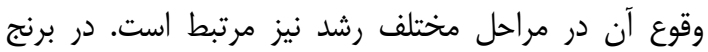

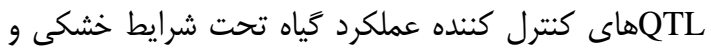

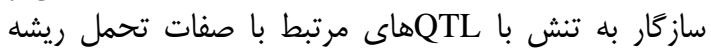

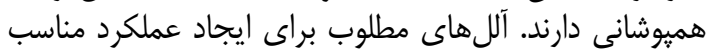

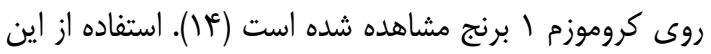

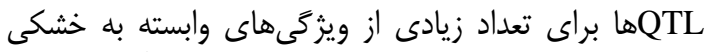

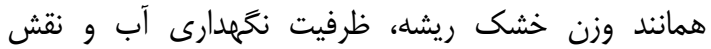

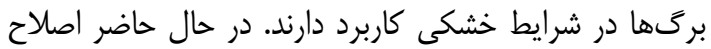

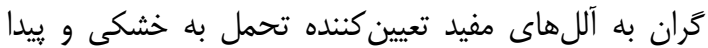

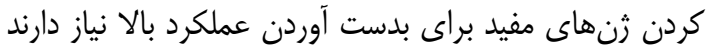

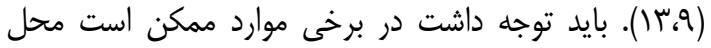

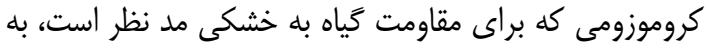

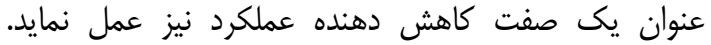

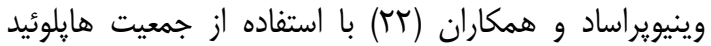

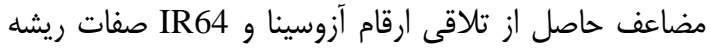

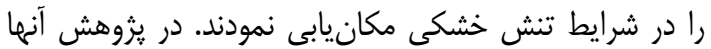

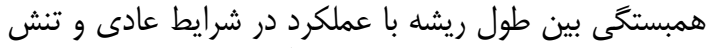

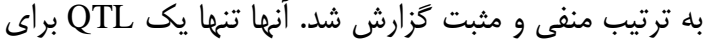

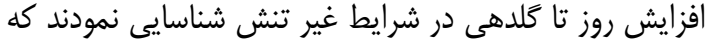


خشكى تحت پِتاسيل اسمزى ه- بار مانيتول قرار كرفتنتد.

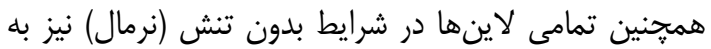

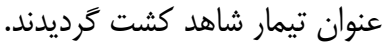

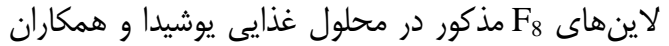

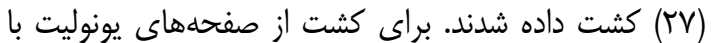

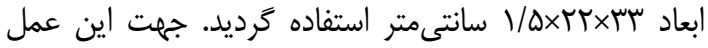

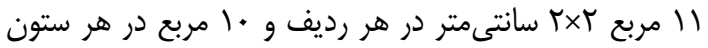

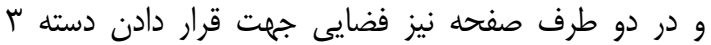

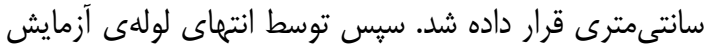

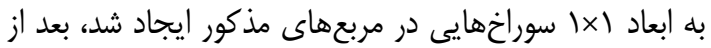

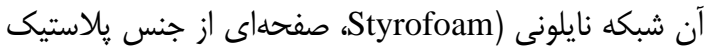

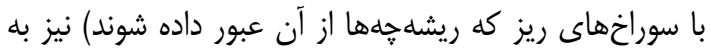

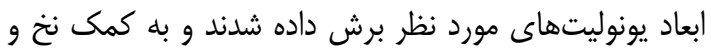

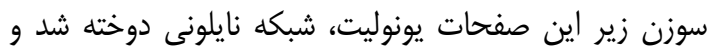

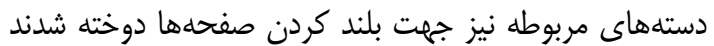

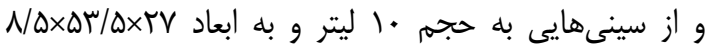

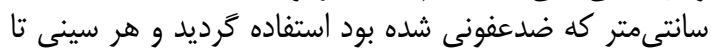

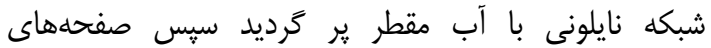

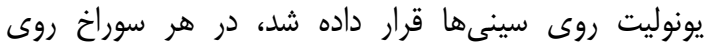

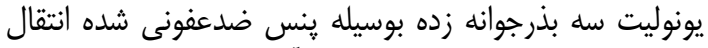

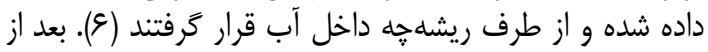

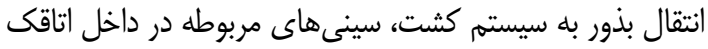

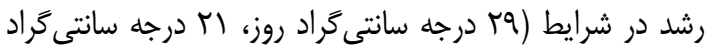

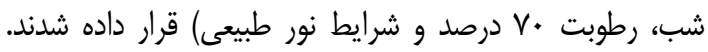

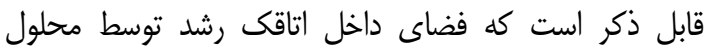

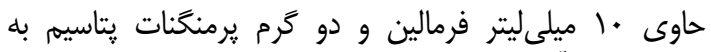

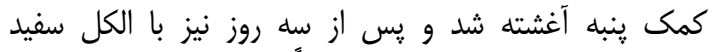

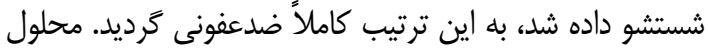

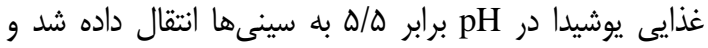

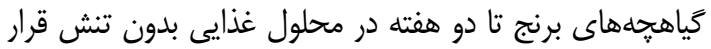

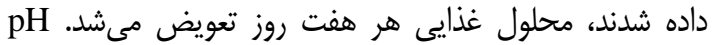

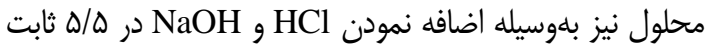

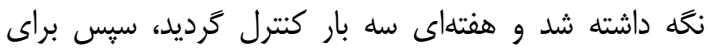

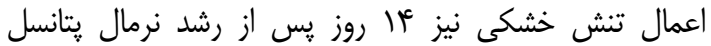

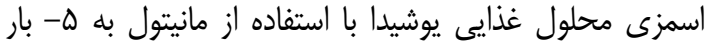

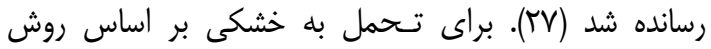

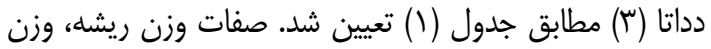

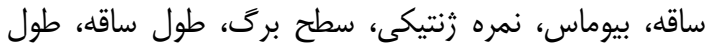

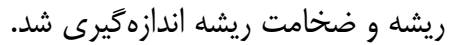

ارقام Bala Azuceana و استفاده نمودند. صفات مربوط به به

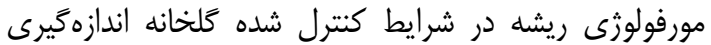

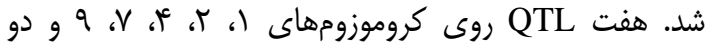

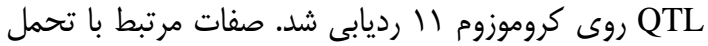

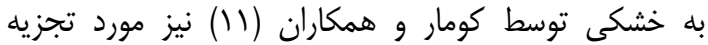

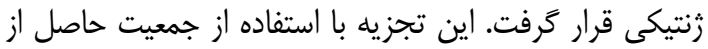
تلاقى

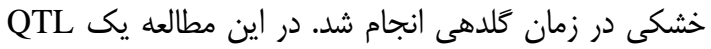

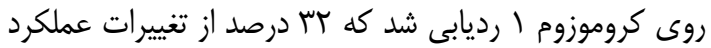

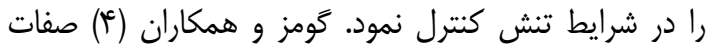

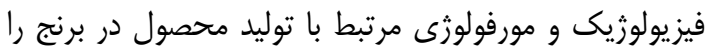

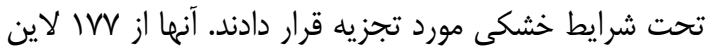

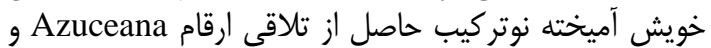

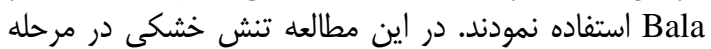

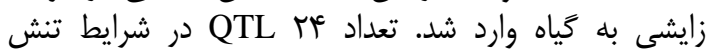

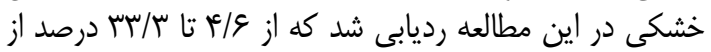

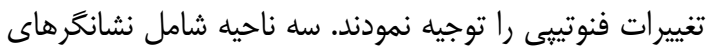

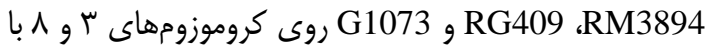

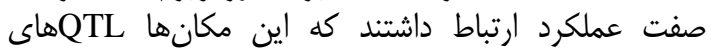

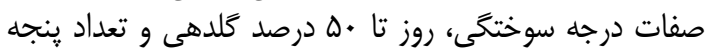

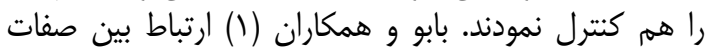

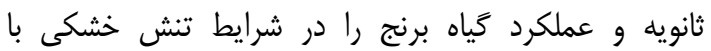

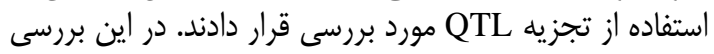

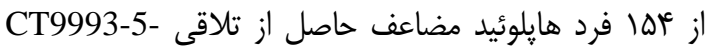

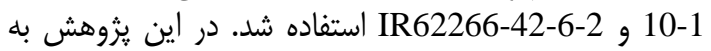

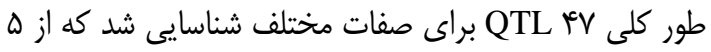

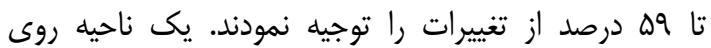

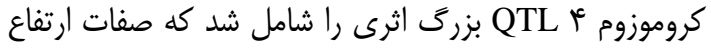
بوته و صفات ريشه را كنترل نمود.

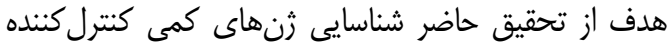

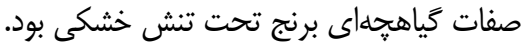

\section{مواد و روشها}

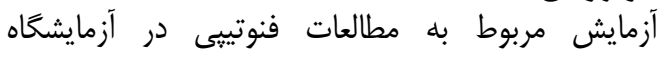

كياهناسى واقع در دانشاءه كنبد كاووس از تاريخ ارديبيهشت

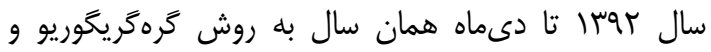

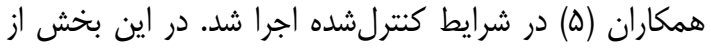

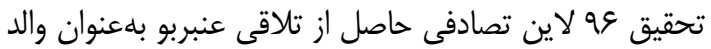

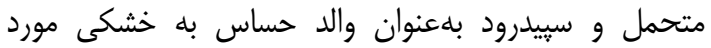

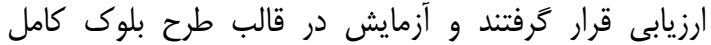
تصادفى در أ تكرار بياده شد. لاينهاى مذكور براى تنش 


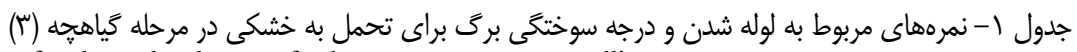
Table 1. Standard evaluation system for drougth tolerance for leave symptoms at seedling stage

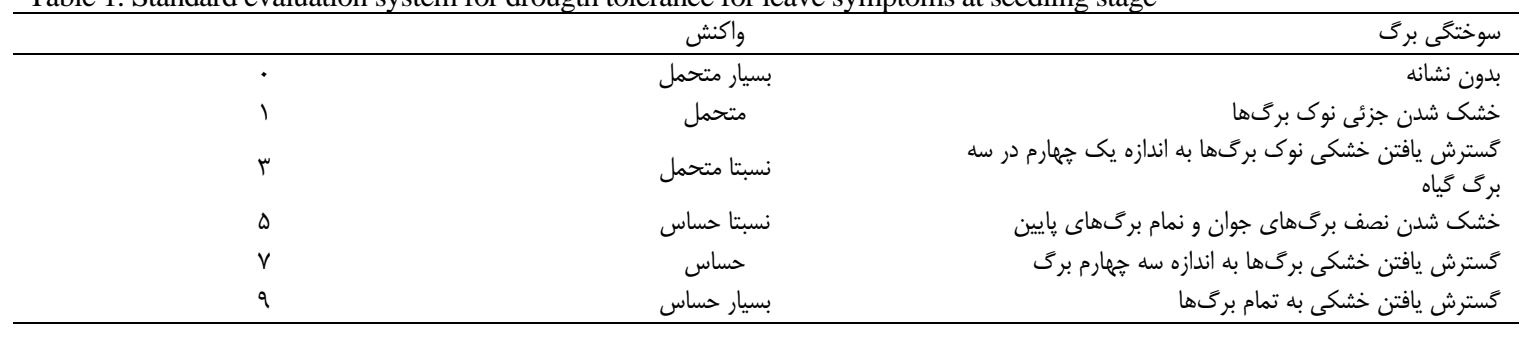

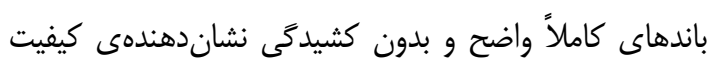

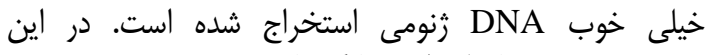

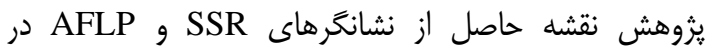

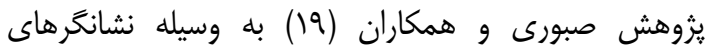

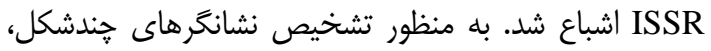

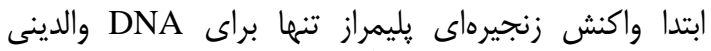

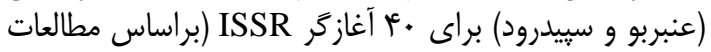

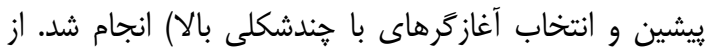

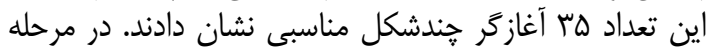

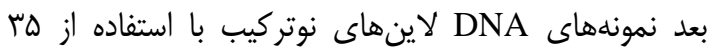
آغازگر جندشكل كه نواربندى واضحترى داشي داشتند، تكثير شدند

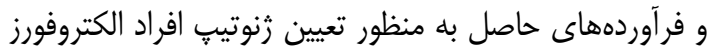
شدند.

Table 2. ISSR primers list

\begin{tabular}{|c|c|c|c|c|c|}
\hline دماى اتصال & 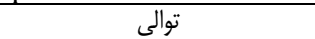 & شماره & دماى اتصال & 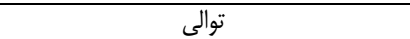 & شماره \\
\hline 59 & AGAGAGAGAGAGAGAGC & MI & 54 & CAGCAGCAGCAGCAG & 1 \\
\hline 59 & GAGGAGAGAGAGAGAGG & tr & 45 & СТСТСТСТСТСТСТСТT & r \\
\hline 53 & GAGAGAGAGAGAGAGAA & re & 42 & ATGATGATGATGATG & f \\
\hline 53 & СТСТСТСТСТСТСТСТT & rQ & 47 & CAACAACAACAACAA & $\Delta$ \\
\hline 48 & СТСТСТСТСТСТСТСТG & ع & 46 & ACTGACTGACTGACTG & $\varepsilon$ \\
\hline 54 & CACACACACACACACAG & r. & 50 & ACACACACACACACACACACTA & 9 \\
\hline 59 & GTGTGTGTGTGTGTGTC & 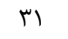 & 52 & ACACACACACACACACACACACG & 1. \\
\hline 59 & GTGTGTGTGTGTGTGTT & זr & 50 & ACAG ACAG ACAG ACAG ACAGC & 11 \\
\hline 54 & ТСТСТСТСТСТСТСТСА & 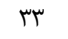 & 52 & CAGCAGCAGCAGCAGCAGA & it \\
\hline 54 & тСТСТСТСТСТСТСТСС & me & 55 & СТССТССТССТССТССТСG & Ir \\
\hline 54 & TGTGTGTGTGTGTGTG & щ & 50 & GTCGTCGTCGTCGTCGTCA & iv \\
\hline 52 & GAATGAATGAATGAAT & rq & 42 & GTTGTTGTTGTTGTTGTTA & ओ \\
\hline \multirow[t]{2}{*}{52} & ССТАССТАССТАССТА & e. & 42 & ТСТTСТTСТTСТTСТTСТG & 19 \\
\hline & & & 40 & TGATGATGATGATGATGAA & $r \cdot$ \\
\hline
\end{tabular}

+ آب دوبار تقطير + بافر بارگذارى) به منظور تعيين اندازه قطعات، ريخته شد.

تجزيه دادهها

جهت تجزيه دادههاى فنوتيبى (تجزيه وادهاريه

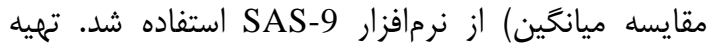

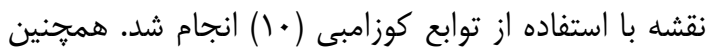

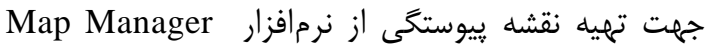

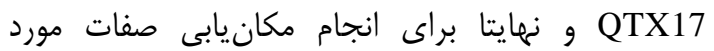

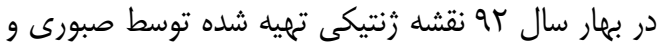

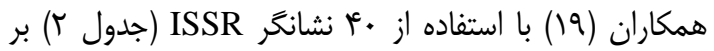

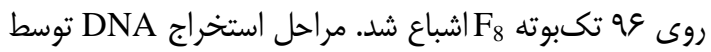

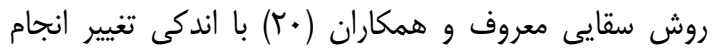

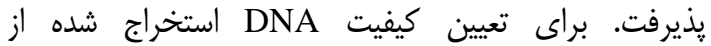
الكتروفورز افقى استفاده شد و از طريق شديت شدت بد باندهاى تشكيل

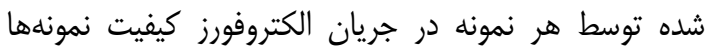

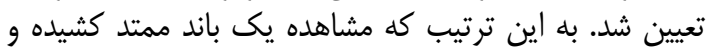

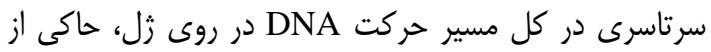

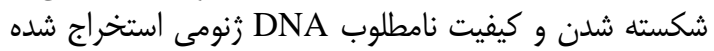

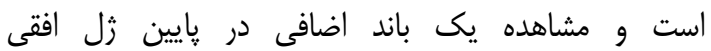
نشاندهندهى وجود RNA در نمونهها مى باشد. مشاهدهى

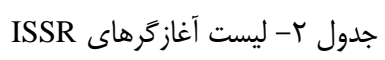

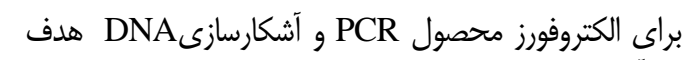

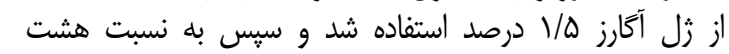

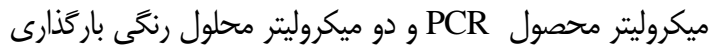

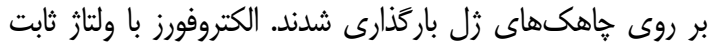

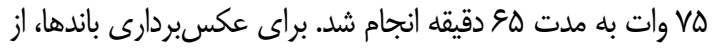

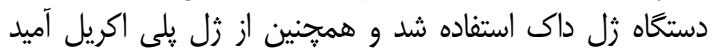

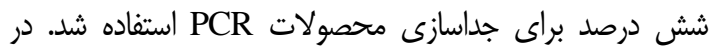

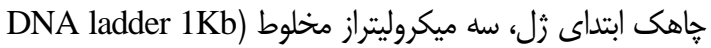


براى صفات ارزيابى شده در مرحله گياهجهاى در شرايط تنش

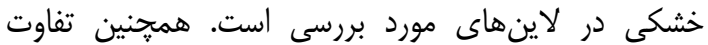

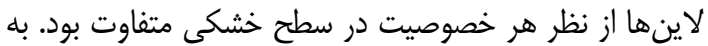

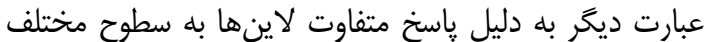
خشكى، اثر متقابل لاين × خشكى ديل معنى دار شد.
بررسى از نرمافزار QTL Cartographer 2.5 استفاده شد.

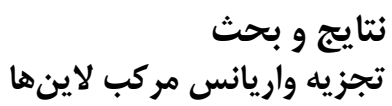

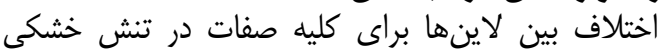

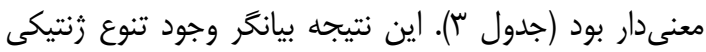

Table 3. Combined Analysis of variance in drought and normal condition

$$
\text { جدول ب- تجزيه واريانس مركب در شرايط نرمال و خشكى }
$$

\begin{tabular}{|c|c|c|c|c|c|c|c|c|c|}
\hline \multirow[b]{2}{*}{ ضخامت } & \multirow[b]{2}{*}{ طول ريشه } & \multirow[b]{2}{*}{ طول ساقه } & \multicolumn{6}{|c|}{ ميانخين مربعات } & \\
\hline & & & سطح برى & نمره زنتيكى & بيوماس & وزن ساقه & وزن ريشه & درجه آزادى & \\
\hline 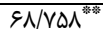 & rqrq/IV/"s.5. & 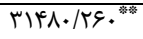 & TEGTY/TIV & $T T V \Delta / T \Delta \Delta^{*}$ & $r \cdot V / \Delta \Delta \zeta^{*{ }^{*}}$ & $r q / T r \omega^{* 6 \%}$ & $\Delta F / \Delta \backslash \Lambda^{* * 6}$ & 1 & محيط \\
\hline . & $F \Delta / T \cdot G$ & $r \| 11 / 98 V$ & $19 / \Delta F$. & $p / \cdot r q$ & س &.$/ \cdot \Delta$. & Tr./. & 9 & خطاى اول \\
\hline 足 & 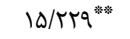 & 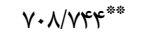 & WN/N\&9" & $\mathrm{r} / \cdot 9 \mathrm{~V}^{\text {券 }}$ & 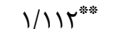 & $\cdot / \Lambda \Lambda r^{* \omega^{*}}$ & 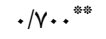 & $9 \Delta$ & محيط × لاين \\
\hline $.1 \cdot 11$ & r/qure & rVQ/זqه & $g / s+4 \mid$ & $\cdot / 41 \wedge$ & r./. & $\cdot 1+r^{c}$ &.$/ \cdot r$. & $\Delta V$. & خطا دوم \\
\hline
\end{tabular}

وزن ساقه و بيوماس بود. همجنين اين لاين داراى كمترين مقدار

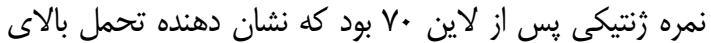

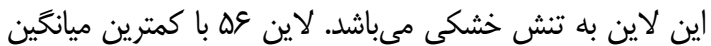

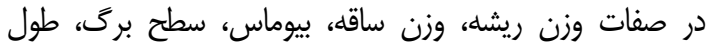

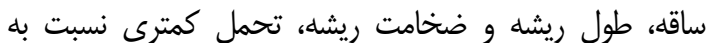

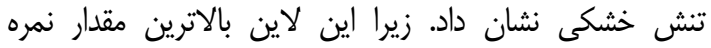

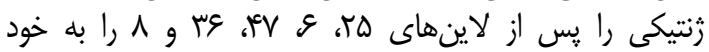

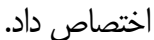

\section{تجزيه واريانس و مقايسه ميانگين لاينها در شرايط} خشكى تهز تجزيه واريانس نشان داد كه اختلاف بين لاينها براى

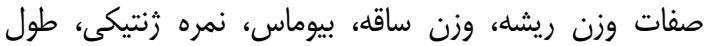

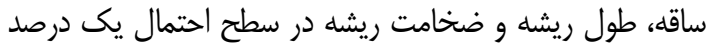

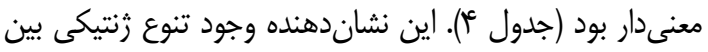

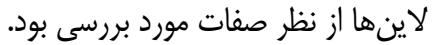
در تنش خشكى لاين سٓ ل داراى بيشترين ميانخين وزن برد ريشه،

Table 4. Analysis of variance in drought condition

جدول ع- تجزيه واريانس ساده در شرايط خشكى

\begin{tabular}{|c|c|c|c|c|c|c|c|c|c|}
\hline & \multicolumn{9}{|c|}{ ميانخين مربعات } \\
\hline ضخامت & طول ريشه & طول ساقه & سطح برى & نمره زنتيكى & بيوماس & وزن ساقه & وزن ريشه & درجه أزادى & \\
\hline 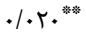 & $\mathrm{V} / \cdot \mathrm{M}^{\mathrm{*} * \mathrm{*}^{*}}$ & $9 \Delta N / \varepsilon \Delta \varepsilon^{n . s}$ & $M / M \Lambda \varepsilon^{* *^{*}}$ & 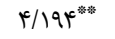 & 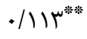 & 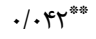 & $\cdot / \cdot r \cdot{ }^{*{ }^{*}{ }^{2}}$ & 90 & لاين \\
\hline$\Delta F / \& \Lambda F$ & Tr/TOV & $V Q / \Delta V T$ & سF/qF & IV/Aץq & IT/VED & ST/VQI & $V / V T^{m}$ & & ضريب تغييرات \\
\hline
\end{tabular}

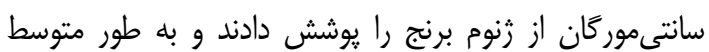

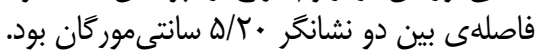

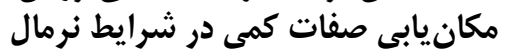

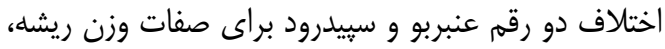

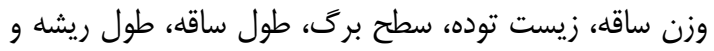

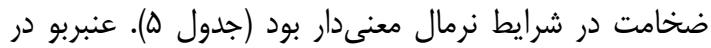
تمامى صفات مقدار بالاترى داشت.
مكانيابى صفات كمى زقئ تمهيه نقشه زنتيكى

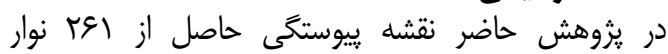
جندشكل AFLP و سז

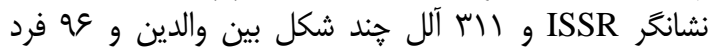

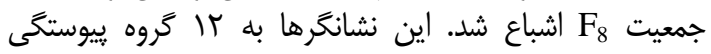

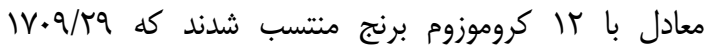


Table 5. Compar mean of traits between parents in normal condition

\begin{tabular}{|c|c|c|c|}
\hline $\mathrm{t}$ & سييدرود & عنبربو & صفت \\
\hline $1 \pi / 9 r^{3}$ & $\cdot / r \Delta \cdot$ & $r / V 99$ & وزن ريشه (كرم) \\
\hline$T / V \in s^{* *^{*}}$ & $\cdot / \mu$. & $\cdot / 4 \Lambda$ & وزن ساقه (كرم) \\
\hline $1 \cdot V r / 94 r^{*}$ & 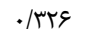 & 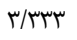 & زيست توده (كرم) \\
\hline$f / q 4 q *$ & $1 \% \%$ & $19 / 0$ & طول ساقه (سانتى متر) \\
\hline 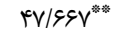 & $\Lambda / \Lambda$ & $11 / \mathrm{V}$ & طول ريشه (سانتىمتر) \\
\hline
\end{tabular}

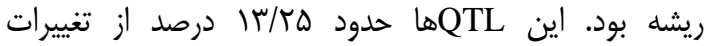

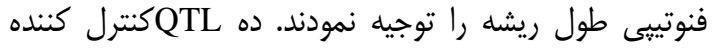

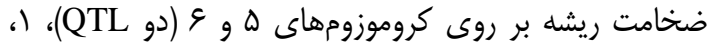

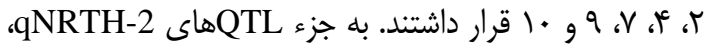

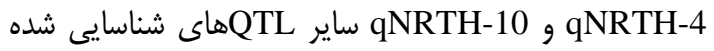

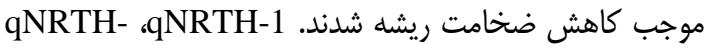

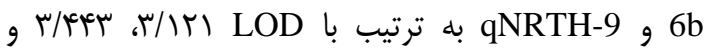

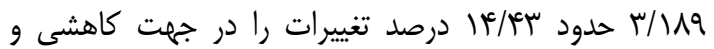

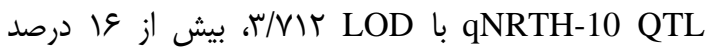

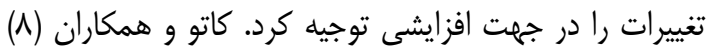

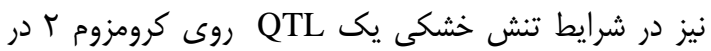

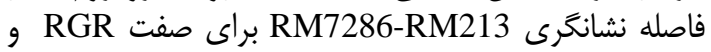

$$
\text { SWU }
$$

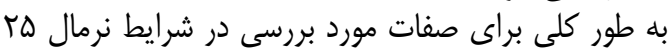

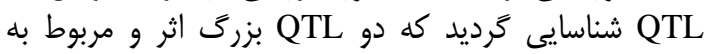

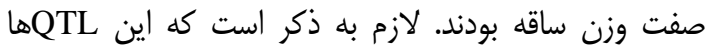

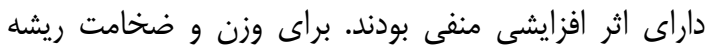

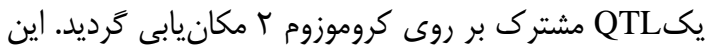

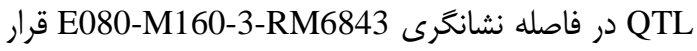

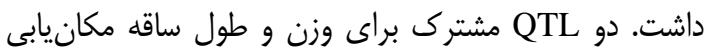

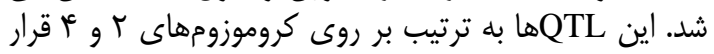

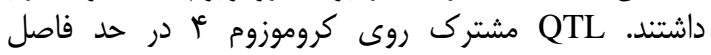
نشانغرهاى RM1359- E070-M150-4 قرار داشت. اين همجنين براى صفت ضخامت ريشه شناسايى

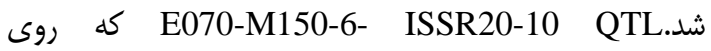
كروموزوم 9 قرار داشت بطور مشترك در صفات طول و ضخامت ريشه مكانيابى شد.

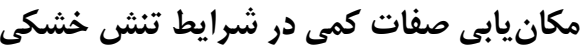

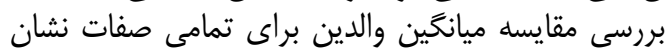

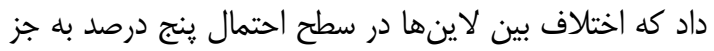

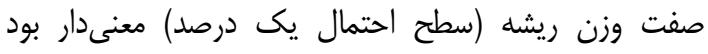

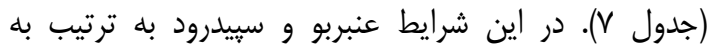
عنوان والد متحمل و تقريبا حساس شناخته شدند.

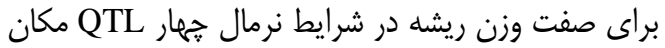

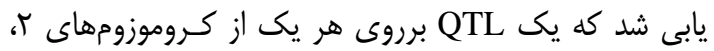

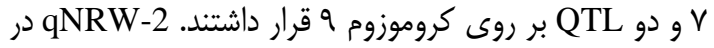

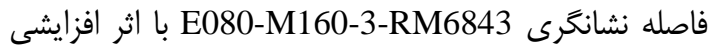

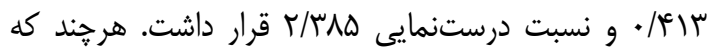

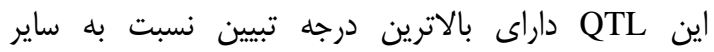

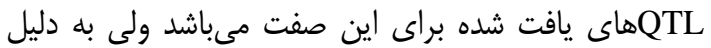

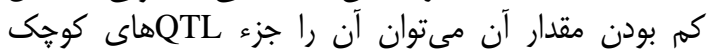

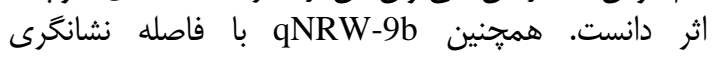
ISSR20-10-E070-M150-7 با اثر افزايشى وهمس/. -- باعث

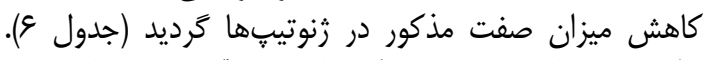

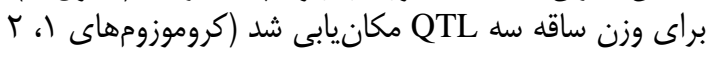

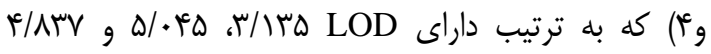
مىباشند. qNSW-2 با فاصله نشانگرى

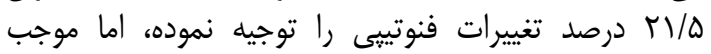

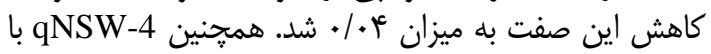

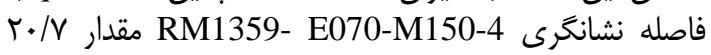

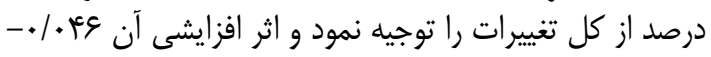

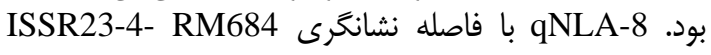

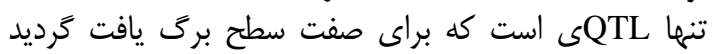

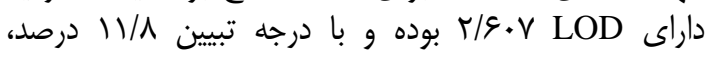

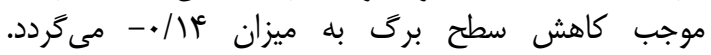

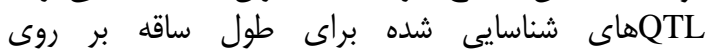

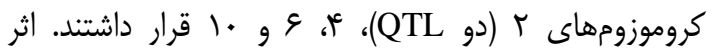

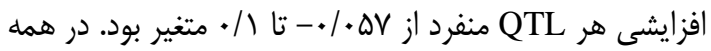

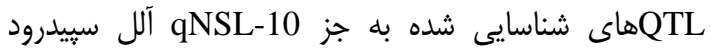

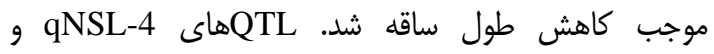

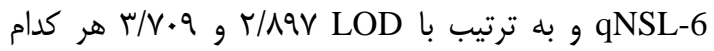

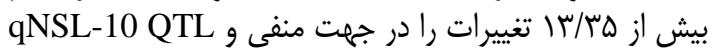

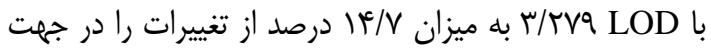

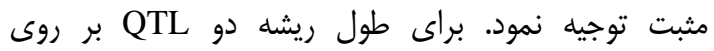

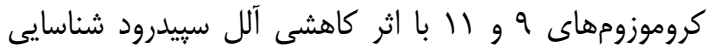

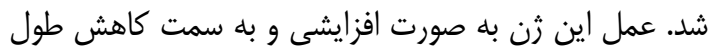


Table 6. Location, LR, additive effects, direction of QTL in normal condition

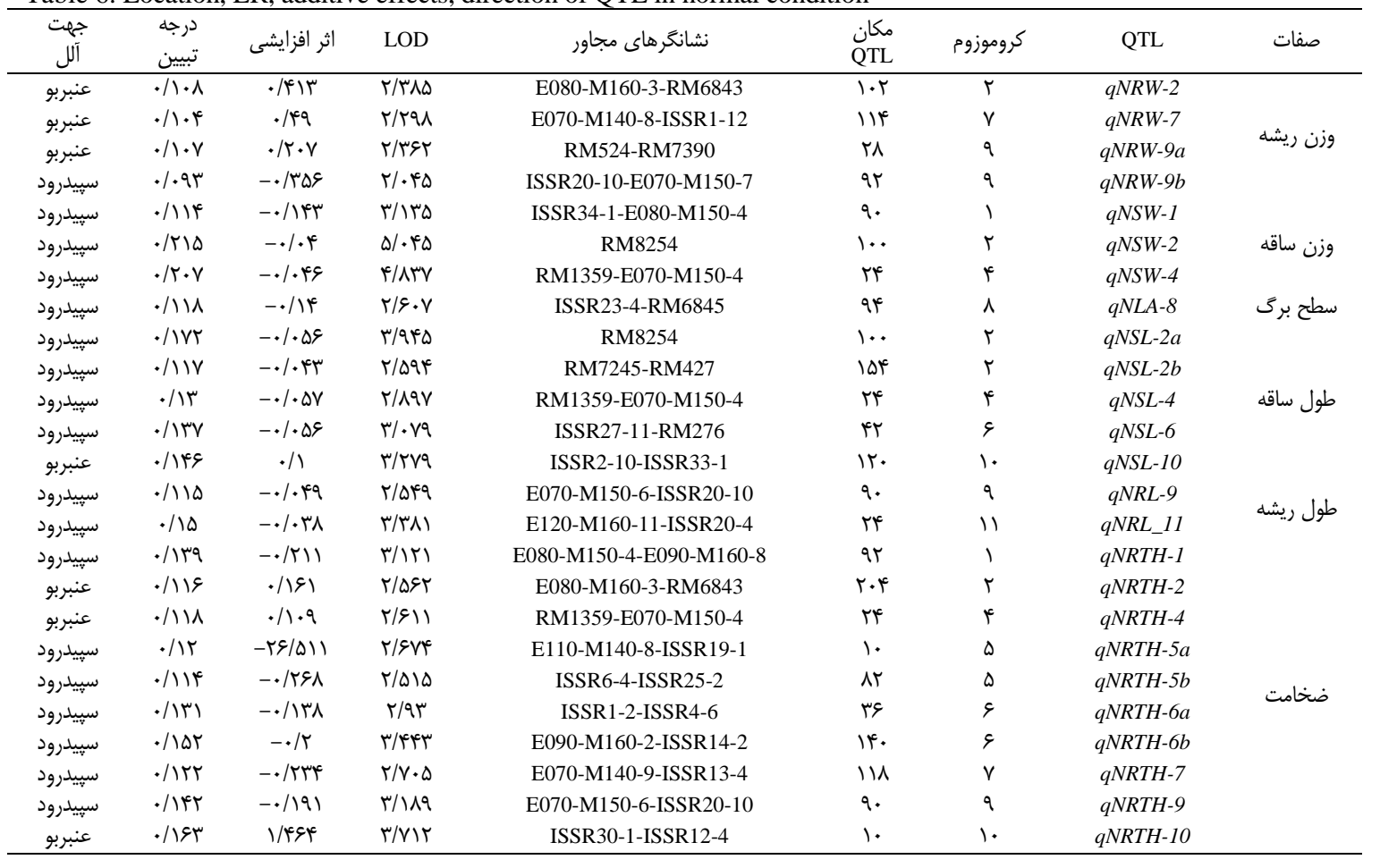

Table 7. Compare mean of traits between parents in drougth condition

جدول V- مقايسه ميانخين صفات مورد بررسى بين والدين در شرايط خشكى

\begin{tabular}{|c|c|c|c|}
\hline $\mathrm{T}$ & سبيدرود & عنبر & صفت \\
\hline$r / G V^{*}$ &.$/ T V$ & $\cdot / r \Delta$ & وزن ريشه (كرم) \\
\hline $\mid r \cdot / r V \cdot * *$ & tat & $1 / \uparrow \wedge$ & وزن ساقه (كَم) \\
\hline r &.$/ 9$ & 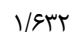 & زيست توده (كرم) \\
\hline 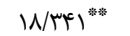 & $\mathrm{v}$ & 1 & نمره زنتيكي \\
\hline $119 \cdot / \cdot \cdot \Delta^{* * *}$ & r/gr| & 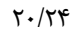 & سطح برگ (سانتى متر مربع) \\
\hline $11 Q / \backslash Q \Lambda^{* * *^{*}}$ & ir & $\Delta F / \Gamma$ & طول ساقه (سانتىمتر) \\
\hline$\Delta S / / A T f^{* * *}$ & $N / 1$ & & طول ريشه (سانتىمتر) \\
\hline 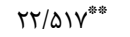 & . &.$/ 19$ & ضخامت (ميلى متر) \\
\hline
\end{tabular}

افزايشى از طرف والد عنبربو توانست س/ با دروصد تغيرات

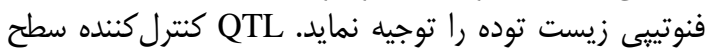

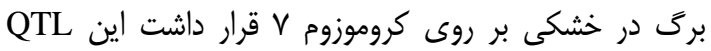
(qDSLA-7)

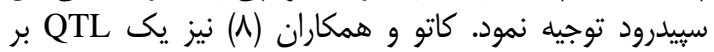

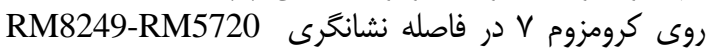
مكانيابى نمودند كه كنترل كننده صفت سرعت روند رشد نسبى كياه

شناسايى شده براى ضخامت ريشه در خشكى بر QTL

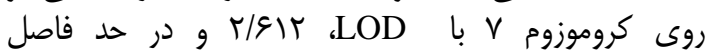

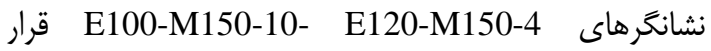

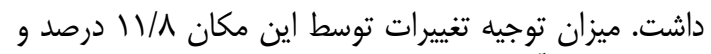

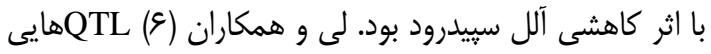

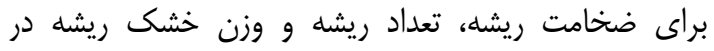

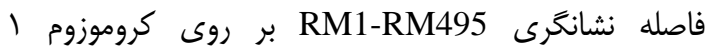

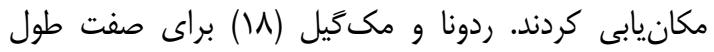

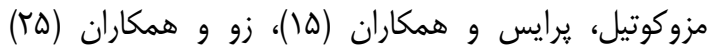

براى وزن ريشه يك QTL بر روى كروموزوم س در حد

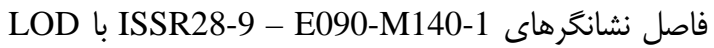

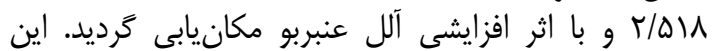

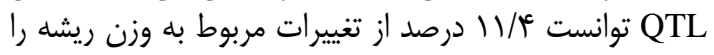

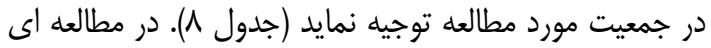

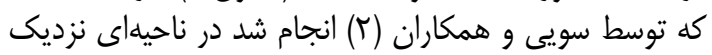

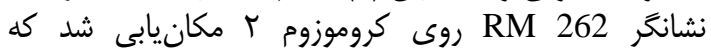

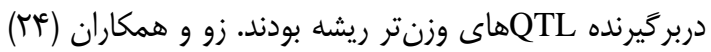

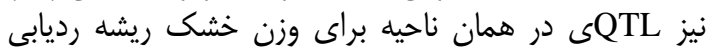

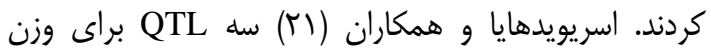

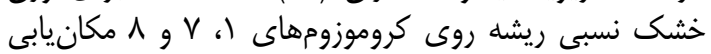

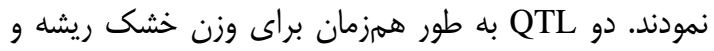

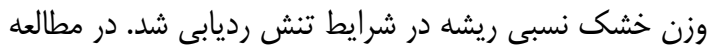

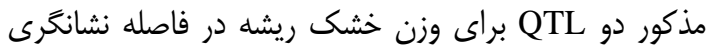

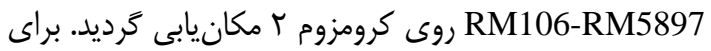

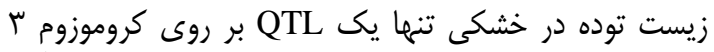

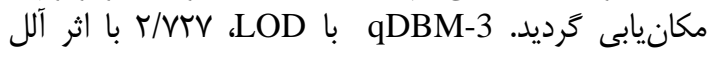


صفات را توجيه نمايد. مقايسه مكانيابى صفات در دو شرايط

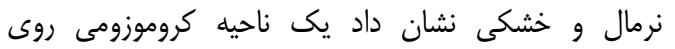

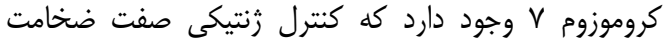

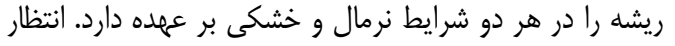

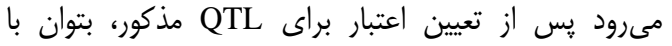

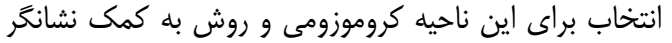

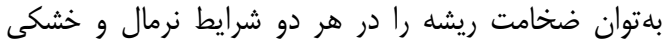
افزايش داد.
S براى طول ساقه و تعداد ريشه در اين ناحيه گزارش نمودند.

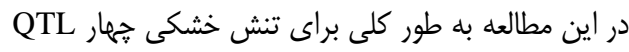

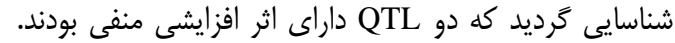

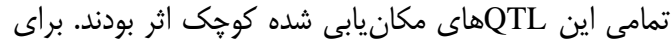
روى صفات وزن ريشه در خشكى و زيست توده يك مانى

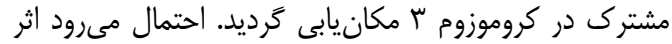
يليوتريويى در اين ناحيه كروموزومى كنترل زئن زنتيكى اين

جدول ^- مكان، نسبت درستنمايى، اثر افزايشى، اثر غالبيت و جهت اثر QTLQ رديابى شده براى صفت خشكى Table 8. Location, LR, additive effects, direction of QTL in normal condition

\begin{tabular}{|c|c|c|c|c|c|c|c|c|}
\hline جهلت & تبيين & افزايشى & LOD & نشانغرهاى مجاور & $\begin{array}{l}\text { مكان } \\
\text { QTL }\end{array}$ & 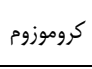 & QTL & صفات \\
\hline عنبربو &.$/ 11 f$ &.$/ \cdot 14$ & $T / \Delta) \Lambda$ & ISSR28-9 -E090-M140-1 & $19 \Lambda$ & r & $q D R W-3$ & وزن ريشه \\
\hline 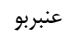 & 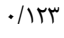 & $\cdot \mid \& \Delta F$ & T/VTV & ISSR28-9-E090-M140-1 & IV. & r & $q D B M-3$ & زيست توده \\
\hline سييدرود &.$/ 14$ & $-1 / 8 \wedge 9$ & $r / 99 V$ & E060-M150-3-RM7179 & $\Delta \xi$ & $\checkmark$ & $q D S L A-7$ & سطح برى \\
\hline سيبدرود &.$/ 111$ & $-1 \cdot 4$ & $r / 9 \mid r$ & E100-M150-10-E120-M150-4 & 8 & $v$ & $q D R T H-7$ & ضخامت ريشه \\
\hline
\end{tabular}

1. Babu, R.C., B.D. Nguyen, V. Chamarerk, P. Shanmugasundaram, P. Chezhian, P. Jeyaprakash, S.K. Ganesh, A. Palchamy, S. Sadasivam, S. Sarkarung, L.J. Wade and H.T. Nguyen. 2003. Genetic analysis of drought resistance in rice by molecular markers: association between secondary traits and field performance. Crop Science, 43: 1457-146.

2. Cui, K., J. Huang, Y. Xing, S. Yu, C. Xu and S. Peng. 2008. Mapping QTLs for Seedling Characteristics under Different Water Supply Conditions in Rice (Oryza sativa L.). Physiologia Plantarum, 132: 53-68.

3. Dedatta, S.K. 1981. Crop establishment technologies and cultural practices for upland rice. Paper presented at the upland rice workshop, $19 \mathrm{pp}$.

4. Gomez, S.M., S.S. Kumar, P. Jeyaprakash, R. Suresh, K.R. Biji, N.M. Boopathi, A.H. Price and R. Babu. 2006. Mapping QTLs linked to physio-morphological and plant production traits under drought stress in rice (Oryza sativa L.) in the target environment. American Journal Biochemistry and Biotechnology, 2: 161-169.

5. Gregorio, G.B., D. Senadhira and R. Mendoza. 1997. Screening rice for salinity tolerance. IRRI. Dis. Paper No. 22, Los Baños. Philippine, 31 pp.

6. Li, Z., P. Mu, C. Li, H. Zhang, Z. Li, Y. Gao and X. Wang. 2005. QTL Mapping of Root Traits in a Doubled Haploid Population from a Cross between Upland and Lowland Japonica Rice in Three Environments. Theoretical and Applied Genetics, 110: 1244-1252.

7. Karimi, H. 1991. Field Crop. Tehran University Press. Third edition, 387 pp.

8. Kato, Y., S. Hirotsu, K. Nemoto and J. Yamagishi. 2008. Identification of QTLs controlling rice drought tolerance at seedling stage in hydroponic culture. Euphytica, 160: 423-430

9. Kordrostami1, M., B. Rabiei, A. Sabouri and H. Sabouri. 2015. Identification of QTLs Controlling Cooking and Milling Quality Traits in an F2:4 Population of Rice (Oryza sativa L.). Journal of Crop Breeding, 7: 16-26 (In Persian).

10. Kosambi, D.D. 1994. The estimation of map distances from recombination values. Annual Eugen, 12: $172-175$.

11. Kumar, R., R. Venuprasad and G.N. Atlin. 2007. Genetic analysis of rainfed lowland rice drought tolerance under naturally-occurring stress in eastern India: Heritability and QTL effects. Field Crops Research, 103: 42-52.

12. MacMillan, K., K. Emrich, H.P. Piepho, C.E. Mullins and A.H. Price. 2006. Assessing the importance of genotype environment interaction for root traits in rice using a mapping population conventional QTL analysis. Theoretical and Applied Genetics, 113: 953-964.

13. Maleki, H.H., N. Abdi, R. Darvishzadeh and M. Jafari. 2016. Mapping QTLs Controlling Drought Tolerance Indices in Sunflower (Helianthus annus L.). Journal of Crop Breeding, 8: 228-235 (In Persian).

14. Norton Gareth, J., M.J. Aitkenhead, F.S. Khowaja, W.R. Whalley and A.H. Price. 2008. A bioinformatic and transcriptomic approach to identifying positional candidate genes without fine mapping: an example using rice root-growth QTLs. Genomics, 92: 344-352.

15. Price, A.H., K.A. Steele, B.J. Moore, P.B. Barraclough and L.J. Clark. 2000. A Combined RFLP and AFLP Linkage Map of Upland Rice (Oryza sativa L.) Used to Identify QTLs for RootPenetration Ability. Theoretical and Applied Genetics, 100: 49-56.

16. Price, A.H., J. Townend, M.P. Jones, A. Audebert and B. Courtois. 2002. Mapping QTLs associated with drought avoidance in upland rice grown in the Philippines and West Africa. Plant Molecular Biology, 48: 683-695. 
17. Price, A.H., K.A. Steele, B.J. Moore and R.G.W. Jones. 2002. Upland rice grown in soil-filled chambers and exposed to contrasting water deficit regimes mapping quantitative trait loci for root morphology and distribution. Field Crops Research, 76: 25-43.

18. Redona, E.D. and D.J. Mackill. 1996. Mapping Quantitative Trait Loci for Seedling-Vigor in Rice Using RFLPs. Theoretical and Applied Genetics, 92: 395-402.

19. Sabouri, H., A. Biabani, M. Katouzi, A. Sabouri, R. Khatami Nejhad, Sh. Mohammad Alegh, M. Najjar, A. and M. Pirasteh. 2011. Relationship between genotype and phenotype in rice under drought stress. Research work in College of Agriculture Science and Natural Resource. Gonbad Kavous University, $145 \mathrm{pp}$.

20. Saghaei Maroof, M.A., R.M. Biyashev, G.P. Yang, Q. Zhang and R.W. Allard. 1994. Extraordinarily polymorphic microsatellites DNA in barely species diversity, chromosomal location and population dynamics. Proceeding of National Academy Science, 91: 5466-5570.

21. Srividhya, A., L.R. Vemireddy, P.V. Ramanarao, S. Sridhar, M. Jayaprada, G. Anuradha, B. Srilakshmi, H.K. Reddy, A.S. Hariprasad and E.A. Siddiq. 2011. Molecular Mapping of QTLs for Drought Related Traits at Seedling Stage under PEG Induced Stress Conditions in Rice. American Journal of Plant Sciences, 2:190-201.

22. Venuprasad, R., H.E. Shashidhar, S. Hittalmani and G.S. Hemamalini. 2002. Tagging quantitative trait loci associated with grain yield and root morphological traits in rice (Oryza sativa L.) under contrasting moisture regimes. Euphytica, 128: 293-300.

23. Vinod, M.S., N. Sarma, K. Manjunatha, A. Kanbar, N.B. Prakash and H.E.S. Hashidhar. 2006. Candidate genes for drought tolerance and improved productivity in rice (Oryza sativa L.). Journal of Bioscience, 31: 69-74.

24. Xu, J.C., J.Z. Li, X.W. Zheng, L. XZou and L.H. Zhu. 2001. QTL Mapping of the Root Traits in Rice Seedling. Acta Genetica Sinica, 28: 433-438.

25. Xu, C.G., X.Q. Li, Y. Xue, Y.W. Huang, J. Gao and Y.Z. Xing. 2004. Comparison of Quantitative Trait Loci Controlling Seedling Characteristics at Two Seedling Stages Using Rice Recombinant Inbred Lines. Theoretical and Applied Genetics, 109: 640-647.

26. Yang, J.C., K. Liu, S.F. Zhang, X.M. Wang, Zh.Q. Wang and L.J. Liu. 2008. Hormones in rice spikelets in responses to water stress during meiosis. Acta Agronomica Sinica, 34: 111-118.

27. Yoshida, S., D.A. Forno, J.H. Cock and K.A. Gomez. 1976. Laboratory manual for physiological studies of rice. IRRI, $83 \mathrm{pp}$. 


\title{
Identification of Genes Controlling Seedling Stage Traits in Iranian Rice Recombinant Lines under Drought Stress Conditions
}

\author{
Mahnaz Katouzi ${ }^{1}$, Saeid Navabpour ${ }^{2}$. Ahad Yamchi $^{3}$, Seyyedeh Sanaz Ramezanpour ${ }^{2}$ \\ and Hossein Sabouri \\ 1, 2 and 3- Graduated M.Sc., Associate Professor and Assistant Professor, Gorgan Agricultural Sciences and Natural \\ Resources University \\ 4- Associate Professor, Gonbad Kavous Universitv (Corresponding Author: hos.sabouri@gmail.com) \\ Received: July 31, $2015 \quad$ Accepted: October 31, 2015
}

\begin{abstract}
Rice is one the most important crops in Iran and worldwide. Abiotic stresses including drought, restrict rice production. In order to saturation of linkage map in recombinant lines population caused by Sepidroud $\times$ Anbarbou crosses, an experiment was conducted using 96 recombinant lines and 40 ISSR markers at Gonbad Kavous University. 96 Recombinant lines were plant under hydroponic conditions for mapping of traits related to drought stress. Shoot weight, root weight, biomass, genetic score, leaf area, shoot length, root length and root thickness were recorded. Linkage map covered $1709.29 \mathrm{cM}$ of rice genome. Four QTLs mapped on chromosomes 3(2 QTLs) and 7 (2QTLs) in drought. qDRW-3 and qDBM-3 with $\mathrm{LOD}=2.518$ and 2.727 had positive additive effects on root weight and biomass, respectively and explained 11.4 and $12.3 \%$ of phenotypic variation. The results of present work can be used for improvement of drought tolerance in rice seedlings after determining marker validation.
\end{abstract}

Keywords: Abiotic stresses, ISSR, Rice, Saturation of linkage map 